\title{
Differential morphine effects on short- and long-latency laser-evoked cortical responses in the rat
}

\author{
Meng-Li Tsai ${ }^{\mathrm{a}}$, Chung-Chih Kuo ${ }^{\mathrm{b}}$, Wei-Zen Sun ${ }^{\mathrm{c}}$, Chen-Tung Yen ${ }^{\mathrm{b}, *}$ \\ ${ }^{a}$ Department of Biomachtronic Engineering, National Ilan University, Ilan, Taiwan \\ ${ }^{\mathrm{b}}$ Institute of Zoology, National Taiwan University, Taipei, Taiwan \\ ${ }^{\mathrm{c}}$ Department of Anesthesiology, National Taiwan University, Taipei, Taiwan
}

Received 1 November 2003; received in revised form 28 April 2004; accepted 10 May 2004

\begin{abstract}
Evoked potential and ensemble neuronal activities were used to study the responses of the primary sensorimotor cortex (SmI) to noxious $\mathrm{CO}_{2}$ laser irradiation of the middle part of the tail in conscious behaving rats. The hypothesis that systemic morphine treatment preferentially attenuates the longer-latency laser-evoked cortical responses was also tested. Laser-evoked potentials (LEPs) and multiple single-unit (SU) activities were, respectively, recorded from chronically implanted stainless-steel screws and microwire electrodes. When examined individually, many SmI neurons showed either short-latency $(<100 \mathrm{~ms})$ or long-latency $(300-500 \mathrm{~ms})$ responses to laser irradiation. These neurons are widely dispersed in the tail region and hind limb region of the SmI, and also in the forelimb and head regions of the primary motor cortex (MI). Quantitatively, a higher percentage of neurons in the SmI tail region responded with shorter latencies compared to those in the SmI hind limb region or in the MI. When responses of many simultaneously recorded SU were examined together, short-latency and longlatency SmI ensemble activities matched the LEP1 and LEP2, respectively. Systemic morphine significantly attenuated the long-latency but not the short-latency component in both LEPs as well as ensemble neuronal activity in the tail region of the SmI. These effects were blocked by naloxone pretreatment.
\end{abstract}

(c) 2004 International Association for the Study of Pain. Published by Elsevier B.V. All rights reserved.

Keywords: Ensemble neuronal activity; Laser-evoked potential; Morphine; Pain; Primary somatosensory cortex

\section{Introduction}

Two major components have been identified from laserevoked potentials (LEPs) recorded in human subjects. Latencies of these potentials respectively correspond to activation of A- $\delta$ and $C$ fibers (Bromm and Treede, 1987; Carmon et al., 1976, 1978; Kentosn et al., 1980). These two LEPs, respectively, coincide with perception of the first (fast or pricking) and the second (slow or burning) pains (Bromm and Treede, 1987). Our recent study showed that following $\mathrm{CO}_{2}$ laser stimulation of the tail of conscious behaving rats, two major cortical potentials (LEP1 and LEP2) were also evoked. Peripheral conduction velocities of LEP1 and LEP2, respectively, correspond to A- $\delta$ and $C$ afferent fiber activation (Kalliomaki et al., 1993; Shaw et al., 1999, 2001). Other evidence that supports LEP1 and LEP2 possibly being

\footnotetext{
* Corresponding author. Tel.: +886-2-3366-2451; fax: +886-2-23625048.

E-mail address: ctyen@ntu.edu.tw (C.-T. Yen).
}

involved in nociception includes a diffuse distribution of these two potential fields over the sensorimotor cortices as demonstrated by multiple channel surface mapping, and the fact that LEP1 and LEP2 are both very sensitive to general anesthesia (Kalliomaki et al., 1993; Shaw et al., 1999, 2001). Although it has been demonstrated with intracortical multi-unit recording that cortical neuronal changes are at least partially responsible for LEP1 and LEP2 (Kalliomaki et al., 1993; Shaw et al., 1999), to our knowledge, the cellular mechanism of LEPs has not been systematically studied. The first objective of the present study was to use a multiple single-unit (SU) recording technique (Nicolelis et al., 1997) to correlate the ensemble neuronal response in the primary sensorimotor cortex (SmI) to laser irradiation compared to the LEP simultaneously recorded in the same rat.

Morphine is one of the most powerful analgesic agents. Psychophysical and behavioral studies, performed on conscious behaving human subjects or monkeys, have consistently shown that morphine preferentially attenuates 
input from unmyelinated nociceptors and reduces second pain (Cooper et al., 1986; Yeomans et al., 1996). In contrast, neurophysiological studies of the central effects of morphine are usually performed on animal subjects under anesthesia. Under anesthetized conditions, morphine has been shown to preferentially affect $\mathrm{C}$-fiber-evoked neural responses in the spinal cord (Carstens et al., 1979; Jurna and Heinz, 1979; Johnson and Duggan, 1981), medulla (Bing et al., 1989), thalamus (Abdulla and Aneja, 1993), and cortex (Kalliomaki et al., 1998). Because of the very strong suppressive effect of general anesthesia on the central nervous system, it is uncertain how neurons at higher levels of the neural axis behave when the animal is awake and behaving. Thus, a gap exists between neurophysiological and the psychological data. The second objective of the present study was to use laser heat-evoked cortical responses of the rat to test the hypothesis that systemic morphine preferentially attenuates LEP2 and the longerlatency cortical ensemble neuronal response in conscious behaving rats. Preliminary data were previously reported (Yen et al., 2002).

\section{Materials and methods}

The entire experimental procedure complied with the proposals of the Committee for Research and Ethical Issues of the International Association for the Study of Pain for laboratory animal use and care and was approved by the Institutional Animal Care and Use Committee, National Taiwan University.

\subsection{Animal preparation and surgical procedures}

Adult female Long Evan rats (250-300 g) were initially anesthetized with sodium pentobarbital $(50 \mathrm{mg} / \mathrm{kg}$, i.p.). Ketamine $(50 \mathrm{mg} / \mathrm{kg}$, i.m.) was administrated as necessary to maintain proper anesthetic depth during surgery. An animal was placed on a stereotaxic apparatus. A midline incision was made. After retracting the skin and cleaning the soft tissue, small craniotomies were made for placement of the intracortical microelectrodes.

Three types of stainless-steel microwire electrodes were used to record SU activity. The first type was a microwire array $(n=6)$ which consisted of 16 Teflon-coated microwires (A-M systems; \#790700). These microwires were aligned in a single file, and the inter-electrode separation of individual microwire was $400-500 \mu \mathrm{m}$ for a total anteriorposterior span of $8 \mathrm{~mm}$, placed parasagittally along the right side of the cerebral cortex about $2-3 \mathrm{~mm}$ lateral to the midline. The microelectrodes were placed $1.5-1.8 \mathrm{~mm}$ deep. The purpose of this type of microelectrode was to record from SUs in the hindpaw and tail region of the SmI, in the primary motor cortex (MI) of the head and forelimb region, and in the occipital cortex (OC) in the same animal. The second type was a smaller linear eight-channel microwire array $(n=6)$. The inter-electrode separation of individual microwire was 200-250 $\mu \mathrm{m}$ for a total anteriorposterior span of $2 \mathrm{~mm}$. They were placed parasagittally along the right side of the cerebral cortex within P2-4 and $\mathrm{R} 2-3 \mathrm{~mm}$, at a final depth of $1.5 \mathrm{~mm}$. The third type was a vertical 8 -microwire bundle $(n=4)$. A thicker Tefloncoated microwire (A-M systems; \#790700) was used as the centerpiece and the ventral-most sensor. Seven formvarinsulated nichrome microwires (A-M systems; \#761000) were glued spirally onto the centerpiece in sequence so that inter-electrode separation of individual microwires was $250-350 \mu \mathrm{m}$ for a total vertical span of about $2 \mathrm{~mm}$. The purpose of the second and the third types of recording microelectrodes was to record many SUs in the tail region of the SmI. To this end, a fine metal microelectrode was used to record from the cortical area first, to ascertain the correct cortical locus. After withdrawal of the test microelectrode, the microwire array or bundle was inserted into the same locus. The final depth of the vertical array electrode was $2 \mathrm{~mm}$. These microwire electrodes were built in-house, and their detailed construction methods were previously published (Tsai and Yen, 2003). The receptive field of each microwire channel was tested by briefly manipulating the body of the rats.

A stainless-steel screw (with an outside diameter of $1 \mathrm{~mm}$ ) was placed in the left side of the skull, at P2.5 and L2.5 mm, as an electroencephalogram (EEG) recording electrode. This was possible because in previous studies, it was shown that tail-evoked potentials can be recorded bilaterally over both sides of the SmI tail region (Shaw et al., 1999, 2001). Reference and ground electrodes were stainless-steel screws of the same kind implanted in the frontal-parietal bones and over the top of the cerebellum (mid-occipital bone), respectively. A pair of three-stranded stainless-steel wires (A-M systems; \#793400) was inserted into the neck muscle to record the electromyogram (EMG). In a few rats, a second pair of EMG recording wires was implanted in the tail flick muscle, the extensor caudae medialis (Brink and Pfaff, 1980), to record the timing and strength of the tail flick behavior. Upon proper placement, the implants were cemented to the animal's skull with dental acrylic.

After completion of the experiment, each animal was deeply anesthetized. Selected recording points of the microelectrode were lesioned electrolytically (50 $\mu \mathrm{A}, 15 \mathrm{~s})$ and perfused transcardially with saline followed by a $4 \%$ formalin solution. Serial $50-\mu \mathrm{m}$ frozen sections of the brain were cut and stained with thionin. The location of the microwire array was determined with microscopic examination of the stained sections (Fig. 1) and documented with camera lucida drawings.

\subsection{Experimental protocol}

After a recovery period of more than 10 days, the animal was transferred to a recording chamber 

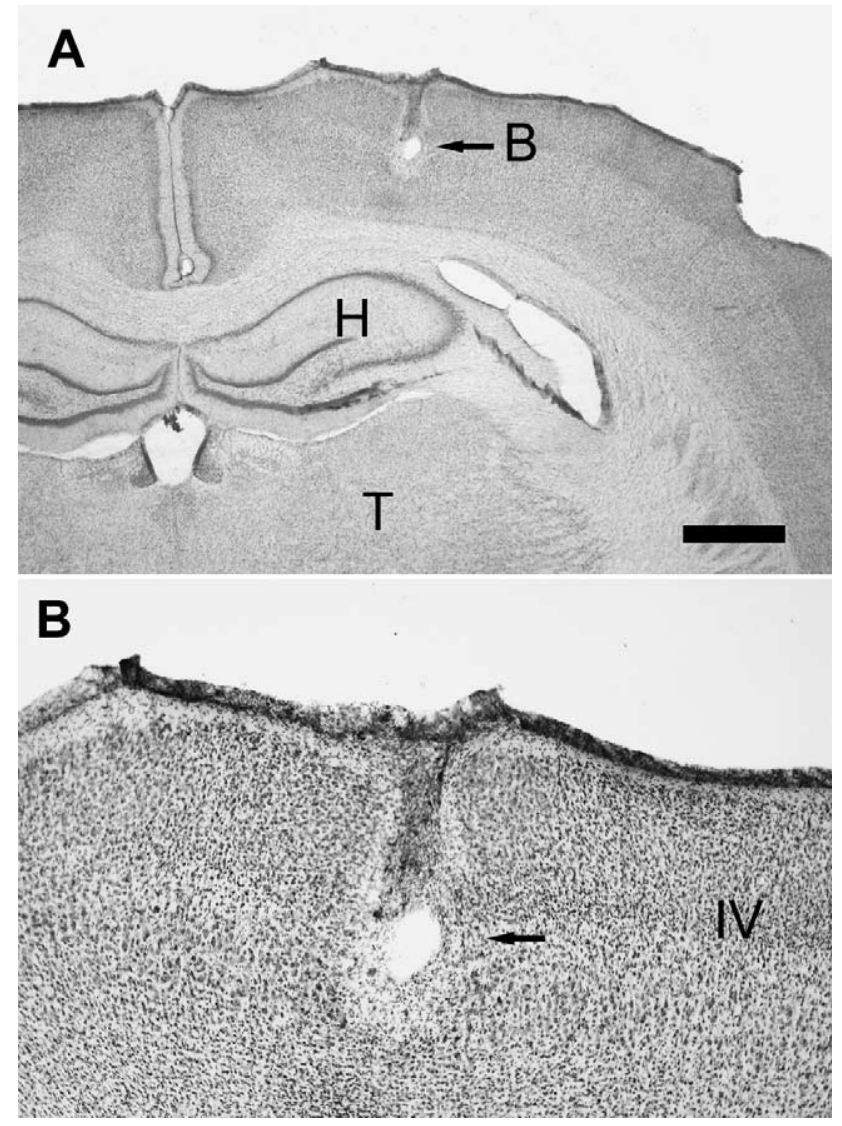

Fig. 1. Photomicrographs showing the low-power (A) and higher-power (B) view of a recording point in the tail representative area of the primary sensorimotor cortex $(\mathrm{SmI})$. An arrow points to the recording point marked with a DC lesion. The eight-channel microwire electrode was implanted parasagittally in the right parietal cortex, and only one channel can be seen in any one transverse section. IV, Layer IV of the SmI; H, hippocampus; $\mathrm{T}$, thalamus. Calibration bar is $1 \mathrm{~mm}$ in (A) and $250 \mu \mathrm{m}$ in (B).

$\left(36 \times 24 \times 30 \mathrm{~cm}^{3}\right.$ in width $\times$ depth $\times$ height, with an open top). To habituate the rat to the experimental chamber, each animal was placed in the same recording chamber five times ( $2 \mathrm{~h}$ /day each) before the experiment. On the day of the actual recording, a 30-min period was allowed for the rat to become familiar with the environment. A head-stage was used to connect the chronically implanted electrodes to a preamplifier whose outputs were sent to a 32-channel MultiNeuronal Acquisition Processor (Plexon, Dallas, TX) for on-line multi-channel spike sorting and acquisition (with a sampling rate of $40 \mathrm{kHz}$ per channel). The extracellular SUs were discriminated in real time using time-voltage windows and a principal component-based template-matching algorithm (SortClient, Plexon). The band-pass filter of the EEG channel was set at $3-90 \mathrm{~Hz}$, with a gain of 5000 . The unit channels were set at $0.5-3 \mathrm{kHz}$, with a gain of 10,000-20,000.

The skin of the middle part of the tail was stimulated with radiant heat pulses emitted from a $\mathrm{CO}_{2}$ laser (medical surgical laser, Tjing Ling \#2, National Taiwan University, $10.6 \mu \mathrm{m}$ wavelength) operating in $\mathrm{TEM}_{00}$ mode (Gaussian distribution) (Yen et al., 1994). The beam diameter was diffused to $3 \mathrm{~mm}$. The duration and the strength of the stimulation pulse were kept at $15 \mathrm{~ms}$ and $8 \mathrm{~W}$, respectively (120 mJ, Shaw et al., 1999, 2001). To minimize tissue damage, sensitization, and habituation, the stimuli were randomly applied to a local skin area on the middle part of the tail $1 \mathrm{~cm}$ in length. The inter-stimulus interval was longer than $10 \mathrm{~s}$. Twenty to thirty laser heat stimulations were made in each session. Jerky movements of the tail under individual stimulation were noted trial by trial. Movements of the tail were further divided into two categories, a strong flick of the tail (tail flick) or a local movement of the tail. The same recording procedures were carried out before the morphine injection (control) and 50 min after drug administration (morphine). Two doses of morphine were administrated to two separate groups of rats (i.p., $10 \mathrm{mg} / \mathrm{kg}, n=9$ and $5 \mathrm{mg} / \mathrm{kg}, n=7$ ). After a recovery period (of at least 2 days), the rat was tested again. Following the control session (post-control), naloxone $(8 \mathrm{mg} / \mathrm{kg})$ was given intraperitoneally, followed by morphine of the same dose (as the first injection several days ago in its respective group) $30 \mathrm{~min}$ after, and the same laser stimulation experiment was repeated another $30 \mathrm{~min}$ after the morphine injection (naloxone + morphine). Naloxone hydrochloride was obtained from Research Biochemical International (Natik, MA), and morphine sulfate was obtained from the Narcotics Bureau, Taiwan.

\subsection{Data analysis}

SU and EEG signals were analyzed with Offline Sorter and Nex (Plexon), Excel (Microsoft), and SigmaPlot (Jandel). Peri-event histograms were generated with a bin size of $10 \mathrm{~ms}$ and a 'boxcar' filter (with a width of two bins) for post-processing. The SmI tail and hindpaw regions were identified by their brisk SU responses being, respectively, restricted only to stimulation of the tail or contralateral paw when the rat was under anesthesia during surgery and the day the animal was sacrificed. MI and OC regions were estimated with stereotaxic coordinates $(2-4 \mathrm{~mm}$ lateral to the midline in both cases, $1-4 \mathrm{~mm}$ rostral to the bregma for the MI and more than $4 \mathrm{~mm}$ caudal to the bregma for the OC, respectively) (Paxinos and Watson, 1998). Multiple SU data were linearly summed to obtain the ensemble neuronal activity of that particular cortical region (see Fig. 4). Ensemble neuronal data (or SU data) within each 10-ms bin were normalized as $Z$ values. This was done by first subtracting the mean values of the baseline activities (i.e. the average activity during the 0.5 -s period preceding the stimuli), and then dividing by the standard deviation of the baseline activities. In order to detect the response pattern of the ensemble neuronal activity (or SU activity), a 99\% confidence level set by the 0.5-s duration before the stimulation was taken as a threshold. Only when spike clusters exceeded this threshold for more than three 
consecutive bins (i.e. $30 \mathrm{~ms}$ ) were the sessions taken as responsive. For a quantitative estimation of the responses corresponding to LEP1, average values of the $30-\mathrm{ms}$ duration including the peak were calculated; as for responses corresponding to LEP2, the average values of bins within the 50-ms juxta-peak duration were used. The 30- and 50-ms durations approximately corresponded to the half-widths of LEP1 and LEP2, respectively. The peak-topeak value was calculated as the quantitative index of LEP1 and LEP2. The LEPs were also normalized to $Z$ values. Paired $t$-tests were used to compare the responses before and after morphine, or before and after combined naloxonemorphine administration. Data are expressed as the mean \pm SE where not specified.

\section{Results}

\subsection{Normal responses to laser irradiation of the mid-tail}

Low intensity, beam-diffused $\mathrm{CO}_{2}$ laser irradiation of the middle part of the tail produced moderate nociceptive responses which included an average of $67 \pm 5 \%$ tail flicks in conscious behaving rats $(n=16)$. The tail flick latency as measured from the tail flick muscle EMG was $350 \pm 9 \mathrm{~ms}$ (Table 1). Two LEPs were clearly discernable from the EEG recordings taken from the skull electrode placed over the left SmI tail region (an example is shown in Fig. 4). The average latencies of the negative peaks of LEP1 and the LEP2 were $66 \pm 3$ and $362 \pm 9 \mathrm{~ms}$, respectively (Table 1).

In all cases, well-isolated SUs were obtained in many of the microwire electrodes. Representative examples of unit sorting and their responsiveness to noxious $\mathrm{CO}_{2}$ laser stimulation are shown in Figs. 2 and 4. In total, 118 wellisolated SUs were recorded in the 16 rats. Individual SUs in the SmI, MI, and OC were responsive either only to the fast component, to both the fast and slow components, or only to the slow component, or were non-responsive. Units were also inhibited after laser irradiation of the tail (data not shown). When units were analyzed according to their functional areas, there was a tendency for higher percentages of non-responsive units in the MI than the SmI. The tail SmI had the highest ratio of units responded to both the fast and the slow components (Fig. 3A). An analysis of the latencies of the responsive units showed that SUs in the tail region of the SmI had significantly shorter latencies than those in the hindpaw region of the SmI and MI (Fig. 3A inset). Five SUs were recorded in the $\mathrm{OC}$, of which two seemed to have a longlatency type of response that did not reach significance, while the other three did not respond at all.

A comparison of the responsiveness of the units in these cortical areas to laser radiant heat can also be appreciated in the combined peri-event histogram shown in Fig. 3B. Units in the SmI tail region had the briskest responses for both the short- and the long-latency components, and units in the SmI hindpaw region had intermediate average response. The pooled MI units also showed both short- and longlatency responses to laser irradiation of the mid-tail, but the responses did not reach a significant level. Also, there was a general increase in spontaneous activities after laser stimuli owing to an increased alertness in all regions tested.

Recordings were successfully carried out in the SmI tail region of all 16 rats. Judging from the ensemble neuronal activities, 12 rats (75\%) exhibited a significant short-latency component and 15 (94\%) exhibited a significant longlatency component. A representative example is shown in Fig. 4. The significantly increased ensemble activity in the short- and long-latency components closely matched the negative peaks of LEP1 and LEP2, respectively. Recordings in the SmI hind paw region were successfully carried out in five rats, among which two rats $(40 \%)$ exhibited a shortlatency component, and three rats $(60 \%)$ exhibited a longlatency component. Among six rats in which recordings were successfully made in the MI region, two rats (33\%) exhibited a short-latency component, and one rat (17\%) exhibited a long-latency component. The ensemble neuronal activities in the $\mathrm{OC}$ of the four rats were not tested for the significance of their responsiveness because of the very small number of units obtained.

The response patterns of LEPs and ensemble neuronal activities of the SmI tail region were further compared. The cortical neuronal responses closely matched the fluctuating LEPs under control, morphine-treated (Fig. 6Aa),

Table 1

Occurrence and latency of SmI single-unit (SU), evoked potential (LEP) and tail flick muscle EMG to $8 \mathrm{~W}, 15 \mathrm{~ms} \mathrm{CO}_{2}$ laser irradiation of the mid-tail of the rat

\begin{tabular}{|c|c|c|c|c|c|c|c|}
\hline & & \multicolumn{3}{|c|}{ No. of responsive/no. of total rats } & \multicolumn{3}{|c|}{ Latency (ms) } \\
\hline & & SU & LEP & EMG & SU & LEP & EMG \\
\hline \multirow{2}{*}{ Control } & Short-latency & $12 / 16$ & $14 / 16$ & $2 / 9$ & $44 \pm 2$ & $66 \pm 3$ & 50,60 \\
\hline & Long-latency & $15 / 16$ & $16 / 16$ & $9 / 9$ & $365 \pm 13$ & $362 \pm 9$ & $350 \pm 9$ \\
\hline \multirow[t]{2}{*}{ Morphine $5 \mathrm{mg}$} & Short-latency & $5 / 7$ & $6 / 7$ & $0 / 3$ & $42 \pm 2$ & $73 \pm 7$ & - \\
\hline & Long-latency & $6 / 7$ & $7 / 7$ & $2 / 3$ & $372 \pm 17$ & $411 \pm 32$ & 350,310 \\
\hline \multirow[t]{2}{*}{ Morphine $10 \mathrm{mg}$} & Short-latency & $6 / 9$ & $9 / 9$ & $0 / 6$ & $46 \pm 2$ & $59 \pm 5$ & - \\
\hline & Long-latency & $6 / 9$ & $7 / 9$ & $2 / 6$ & $347 \pm 7$ & $329 \pm 5$ & 320,320 \\
\hline
\end{tabular}


A

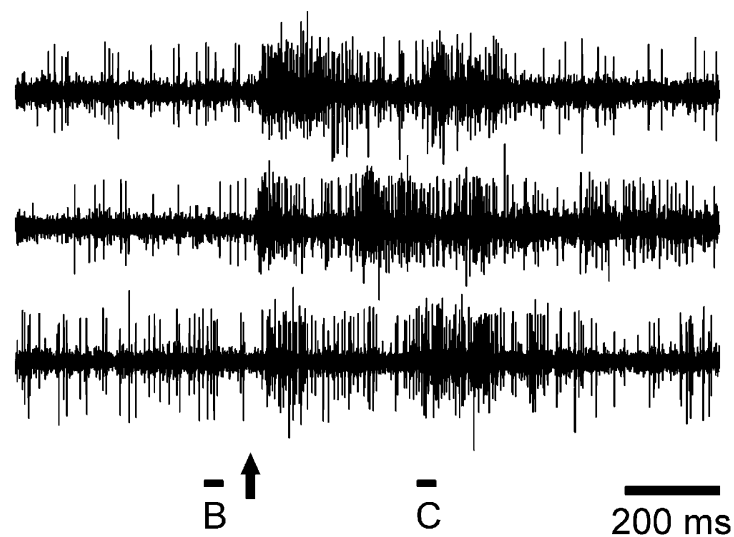

B
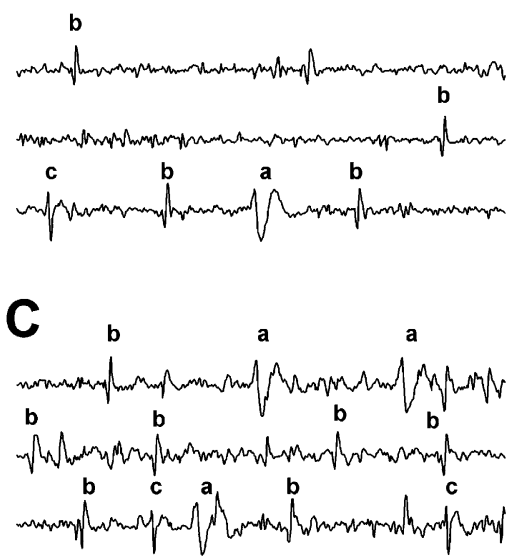
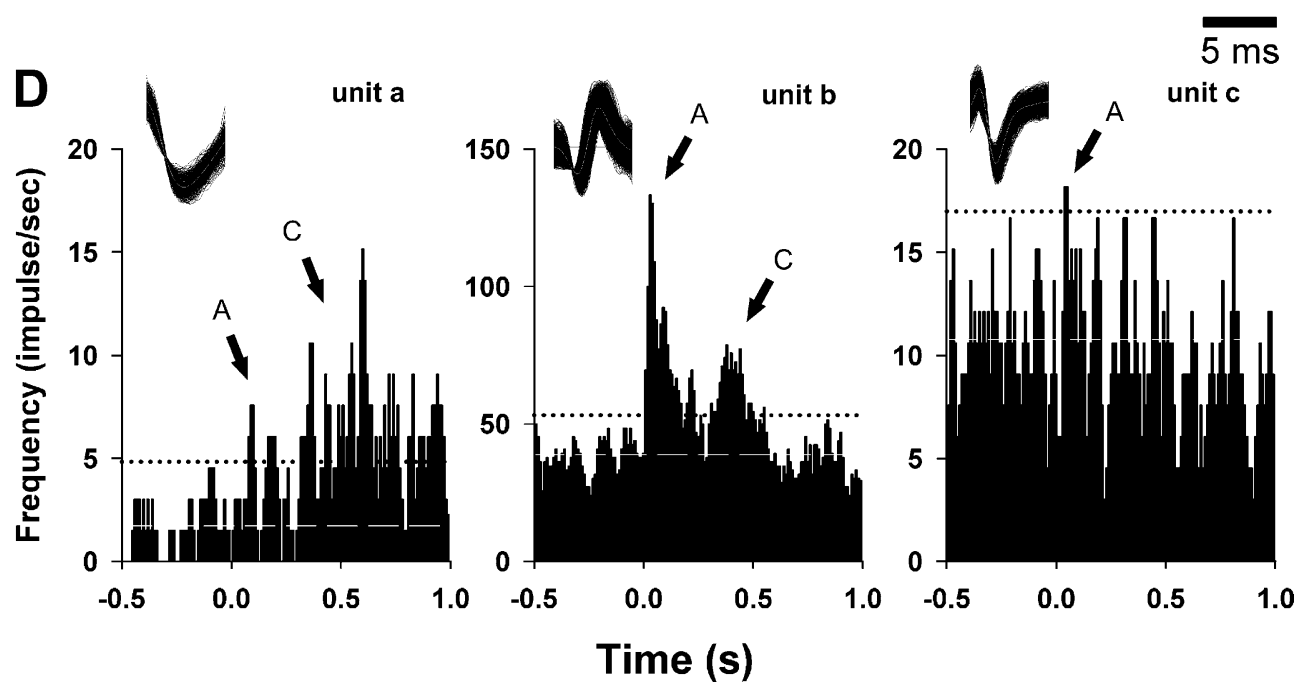

Fig. 2. Representative examples of cortical neuronal responsiveness to noxious heat stimulation of the mid-tail region by $\mathrm{CO}_{2}$ laser. On the upper row are original traces recorded from a single microwire (A). The arrow points to the time the laser pulse (15 ms, $8 \mathrm{~W})$ was given. Regions B and C are expanded in (B) (before laser stimulation) and (C) (after laser stimulation). Three SUs were isolated from these traces. They were labeled as units a, b, and c. The peri-event histograms of units a, b, and c to 22 laser pulses are shown in (D) from left to right, respectively. Bin width: 10 ms. Insets are the superimposed waveforms of the three units in the 280 -s recording period. There were 565 spikes for unit a, 11574 spikes for unit b, and 2512 spikes for unit c. The dotted white lines in the figures are average baseline activity (in the $0.5 \mathrm{~s}$ preceding the laser pulses), and the dotted lines are $99 \%$ confidence lines estimated from the baseline activity. Arrows $\mathrm{A}$ and $\mathrm{C}$ point to the fast and the slow unit responses, respectively.

post-control, and naloxone/morphine-treated conditions (Fig. 6Ab).

\subsection{Morphine's effect on LEP and cortical ensemble activities}

Two morphine dosages were used. Intraperitoneal injection of $5 \mathrm{mg} / \mathrm{kg}$ morphine $(n=7)$ suppressed the strong tail flick response of the rats to $17 \pm 10 \%$ (suppressed to $25 \%$ of control, Fig. 5B). In another group of rats treated with a higher morphine dosage $(10 \mathrm{mg} / \mathrm{kg}$, $n=9$ ), the tail-flick of the rat was totally suppressed $(1 \pm 1 \%), 50 \mathrm{~min}$ after drug administration. There were parallel progressive attenuations of the long-latency ensemble SU response and LEP2 (Fig. 5). The ensemble SU activity seemed to be a more-sensitive index. At the lower dosage of $5 \mathrm{mg} / \mathrm{kg}$, the long-latency ensemble SU response showed significant attenuation (a 37\% decrease, $P<0.05$, by paired $t$-test compared with the control); whereas for LEP2, although a decrease was seen, it did not reach a significant level. At the higher-dose morphine treatment $(10 \mathrm{mg} / \mathrm{kg}$, Figs. 5 and $6 \mathrm{Ba})$, the average LEP2 dropped $38 \%(P<0.05, n=9)$, and the long-latency ensemble SU response dropped $41 \%(P<0.01, n=9)$. In contrast, neither the short-latency ensemble SU response nor LEP1 significantly changed with either morphine dose (Figs. 5 and 6). All these changes were reversible and could be prevented by pretreatment with $8 \mathrm{mg} / \mathrm{kg}$ of naloxone given intraperitoneally $(10 \mathrm{mg} / \mathrm{kg}$ data in Fig. $6 \mathrm{Bb}, 5 \mathrm{mg} / \mathrm{kg}$ data not shown).

The responses of different types of SUs to the two dosages of morphine treatments were examined. LEP2 in the tail region of the $\mathrm{SmI}$ could be generated by $\mathrm{A}^{+} \mathrm{C}^{+}$or $\mathrm{A}^{0} \mathrm{C}^{+}$neurons. The long-latency components of these two 
A

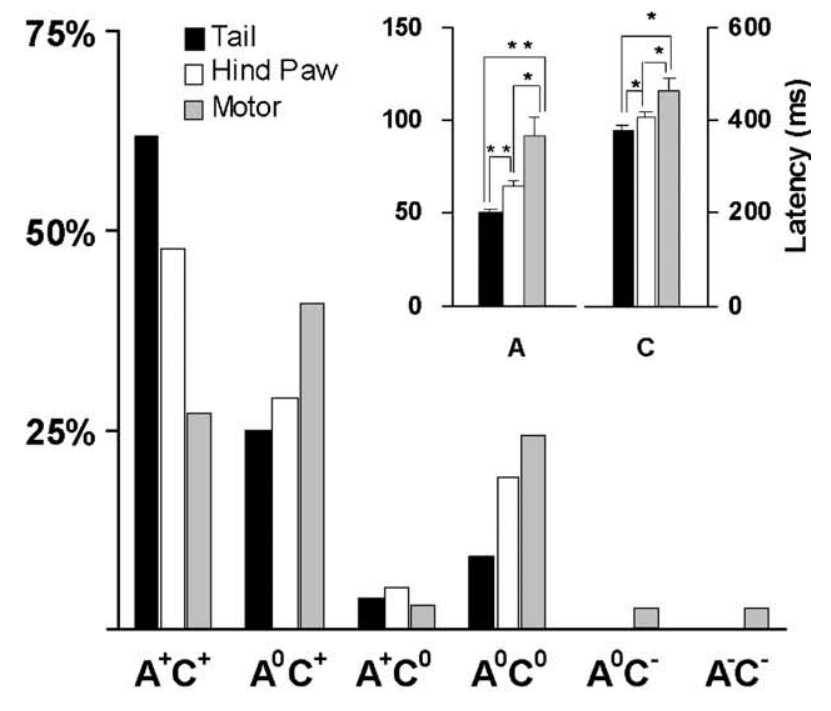

B

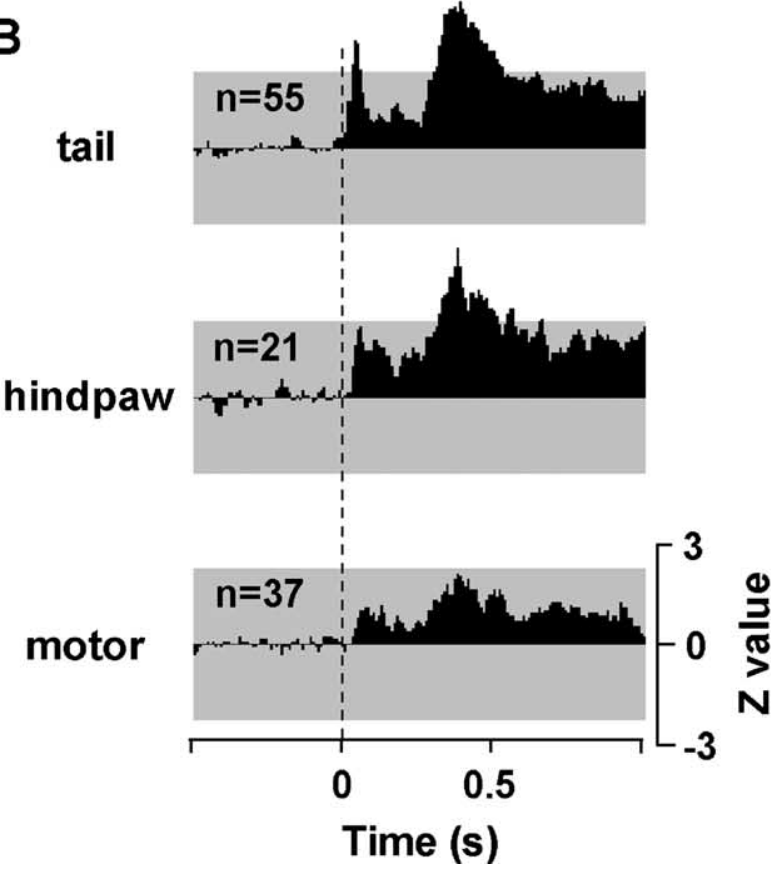

Fig. 3. Summary of single-unit responses to $\mathrm{CO}_{2}$ laser irradiation of the mid-tail. (A) Percentage and peak latency of single-unit responses to $\mathrm{CO}_{2}$ laser stimulation. A: Short-latency response; $\mathrm{C}$ : long-latency response; + , excitatory; - , inhibitory; 0 , not responsive. The tail region of the primary sensorimotor cortex (SmI) showed more shortlatency excitations and more responsive units than those in the other regions. In the inset is a statistical comparison of the latencies of the short-latency response (left panel) and long-latency response (right panel) in the three regions (SmI tail region, filled bar; SmI hind paw region, open bar; and MI, gray bar). Asterisks represent a significant difference $(* * P<0.01 ; * P<0.05)$. (B) Peri-event histogram of wellisolated single-unit responsiveness to $\mathrm{CO}_{2}$ laser irradiation of the midtail. The SUs are grouped into their functional regions, which included the SmI tail region (tail), SmI hind paw region (hind paw), and primary motor cortex region (motor). The units in the SmI tail region had the briskest responses for both the short- and the longlatency components. All data were normalized to $Z$ values of the $0.5 \mathrm{~s}$ preceding the stimuli. Grayish areas are the $99 \%$ confidence zone. Bin width: $10 \mathrm{~ms}$. channel 1

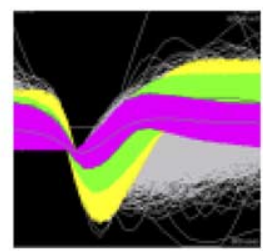

$$
(\boldsymbol{n}+\boldsymbol{n}+\boldsymbol{n}+\boldsymbol{n}) \div 5
$$

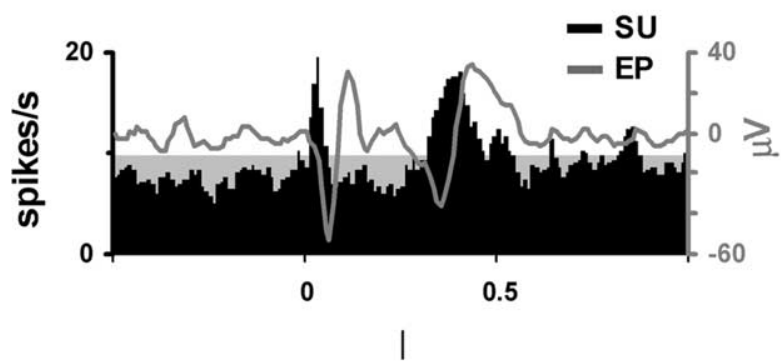

normalized as $Z$ values

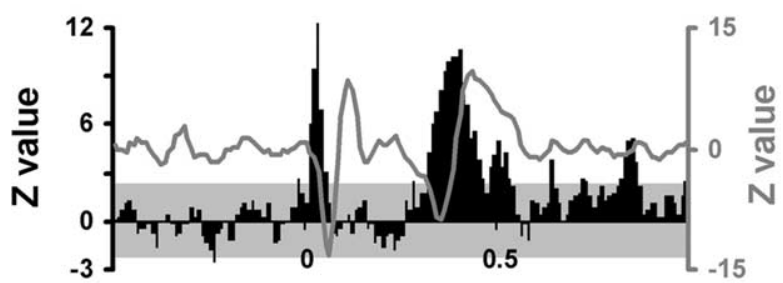

Time (s)

Fig. 4. A representative ensemble neural response of the SmI tail region to laser irradiation of the mid-tail. The ensemble neuronal activity was a linear averaging of the activities of five SUs recorded from two microwire electrodes (channels 1 and 2) whose receptive fields included the tail of the rat. The top insets are superimposed color-coded spike wavelets. Total recording time was $679 \mathrm{~s}$; unit 1a, 4512 spikes; unit 1b, 7790 spikes; unit 1c, 10,953 spikes; unit 2a, 4175 spikes; and unit 2b, 1240 spikes. Peri-event histograms in the middle and lower panels are averaged from 30 laser pulse stimuli. Note the close match between the ensemble single-unit (SU) response and the evoked potential (EP) recorded from a stainless-steel screw electrode implanted in the skull overlying the contralateral tail region of the SmI.

types of neurons did not show preferential sensitivity. They were suppressed by either dosage.

\section{Discussion}

Taking advantage of the fact that laser heat irradiation of the tail produces short- and long-latency cortical evoked potentials in conscious behaving rats, we demonstrate in this study that morphine preferentially suppresses the longlatency evoked potential. This is further supported by ensemble neuronal data which were simultaneously recorded. The morphine effects were reversible and could be prevented by naloxone pretreatment. Our data are also 

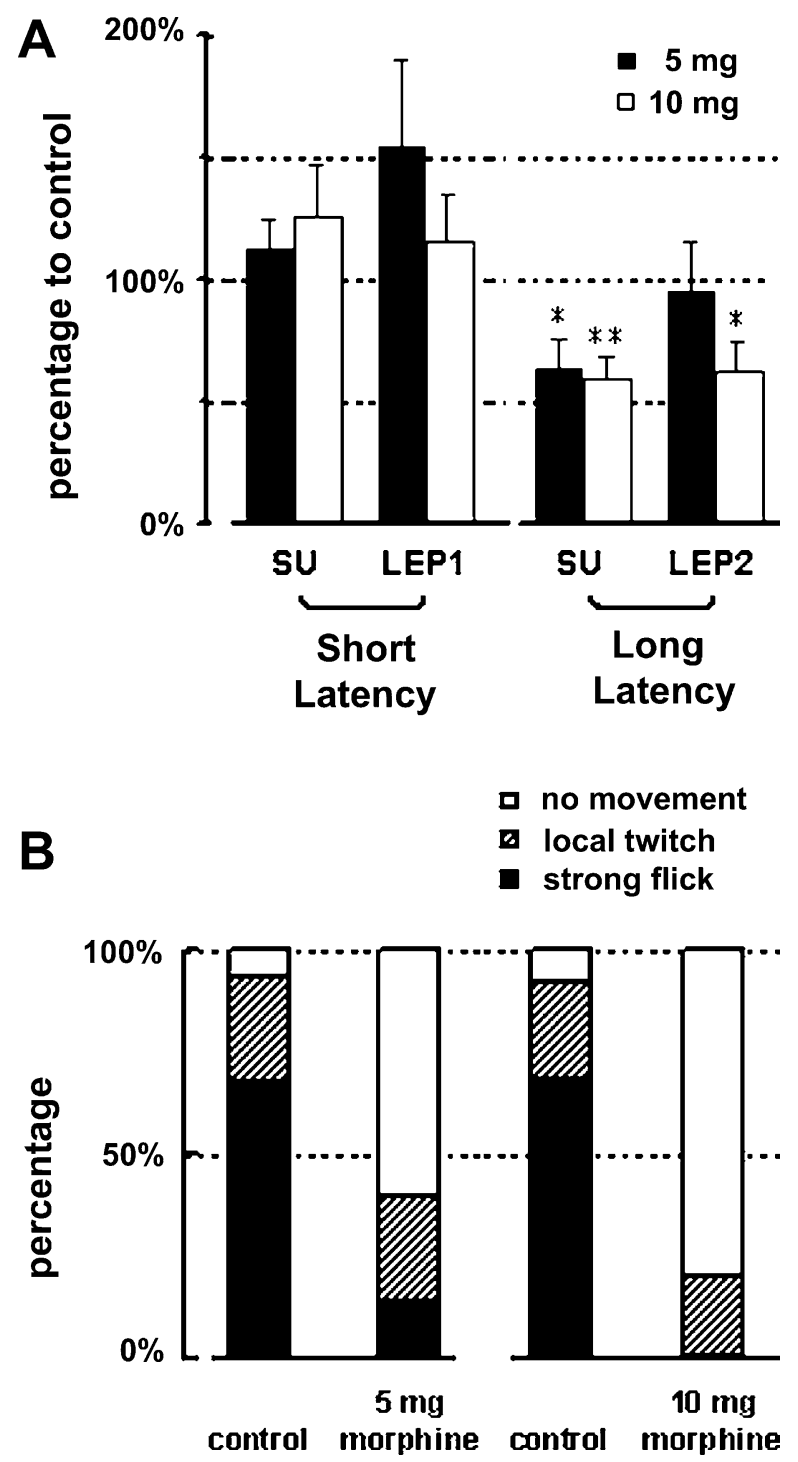

Fig. 5. Dose-response effect of two morphine treatments ( 5 and $10 \mathrm{mg} / \mathrm{kg}$ ) on laser heat-evoked responses of ensemble single-unit activities (SU), evoked potentials (EP), and tail flick in the sensorimotor cortex (SmI) tail region. Note the progressively stronger attenuation of long-latency SU and LEP2 responses and tail flick behavior with the larger morphine dosage $(10 \mathrm{mg} / \mathrm{kg}) . * P<0.05 ; * * P<0.01$.

significant in regard to the cellular basis of LEPs and have possible implications for the functional role of the primary somatosensory cortex (SI) in pain function.

\subsection{Cellular basis of laser-evoked potentials}

The $\mathrm{CO}_{2}$ laser has been a useful tool in pain research since the late 1970s (Mor and Carmon, 1975). It selectively and synchronously activates cutaneous thermonociceptors which conduct in the $\mathrm{A}-\delta$ and $\mathrm{C}$ fiber ranges. In human subjects, the evoked potentials obtained from scalp EEG electrodes are termed 'late' and 'ultra-late', respectively. Cortical evoked potentials related to A- $\delta$ and $C$ fiber conduction velocities have been recorded from the cerebral cortex of the rat. This is first done in anesthetized rat by stimulating different proximo-distal locations of the hind leg (Kalliomaki et al., 1993), and again demonstrated in conscious rat by irradiating the tip, the middle part and the base of the tail (Shaw et al., 1999). They were, respectively, termed LEP1 and LEP2. Both LEP1 and LEP2 are very sensitive to anesthesia. Multiple-channel surfacemapping studies have revealed that LEPs are diffusely distributed over the sensorimotor cortices. That cortical neuronal change is responsible for at least part of these surface recorded cortical potentials has been demonstrated with intracortical multi-unit recordings (Kalliomaki et al., 1993; Shaw et al., 1999). The present study presents the first SU evidence. We show that a high percentage of neurons in the homotopic region of the SmI responded to noxious thermal stimuli: some at shorter A- $\delta$-range latency, some at longer C-range latency, and some at both latencies. Hence, when the activities were spatially summated, ensemble SmI neuronal activity consistently showed both short- and longlatency peaks. In addition, the peaks of the ensemble activities matched the negative peaks of LEP1 and LEP2, respectively.

Laser heat evoked short- and long-latency unit responses, and LEP1 and LEP2 in the rat SmI. These responses significantly differed from A- $\beta$ tactile responses in the SmI by their peripheral conduction velocities, diffusely distributed topography over the cerebral cortex, and their sensitivity to pentobarbital anesthesia (Shaw et al., 1999, 2001) and to morphine treatment (Kalliomaki et al., 1998). It is unclear, however, how much of these responses were direct responses to peripheral nociceptor activation, and how much should be attributed to secondary responses to tail flick movement or other nocifensive behaviors, since the animals were conscious and exhibiting many behaviors. An additional analysis was performed to clarify the possible input sources of the short- and long-latency responses of the SmI.

We noted that under the $5 \mathrm{mg} / \mathrm{kg}$ dose of morphine, the rat moved its tail about half of the time (Fig. 5B). In Fig. 7, the peri-event (laser heat) histogram of the tail SmI units was divided into 'movement' and 'no movement' groups and separately analyzed. Note that in cases where no apparent movement occurred, prominent short- and longlatency SmI unit responses remained in the same latency range (compare the top left and lower left panels). These data show that primary sensory inputs evoked by laser heat were responsible for a significant portion of the short- and long-latency SmI neural responses.

Cortical neurons responsive to laser heat were not restricted only to the homotypic SmI. Thus, laser irradiation of the mid-tail also excited many SmI hindpaw neurons, MI neurons of the forelimb and head region, and even neurons in the OC. These heterotypic regions, however, had lower percentages of responsive neurons (Fig. 3A). The responsive neurons in these regions were less responsive in that their latencies were longer, and their response strength was weaker (Fig. 3B). We deduced that the widely 
(a)

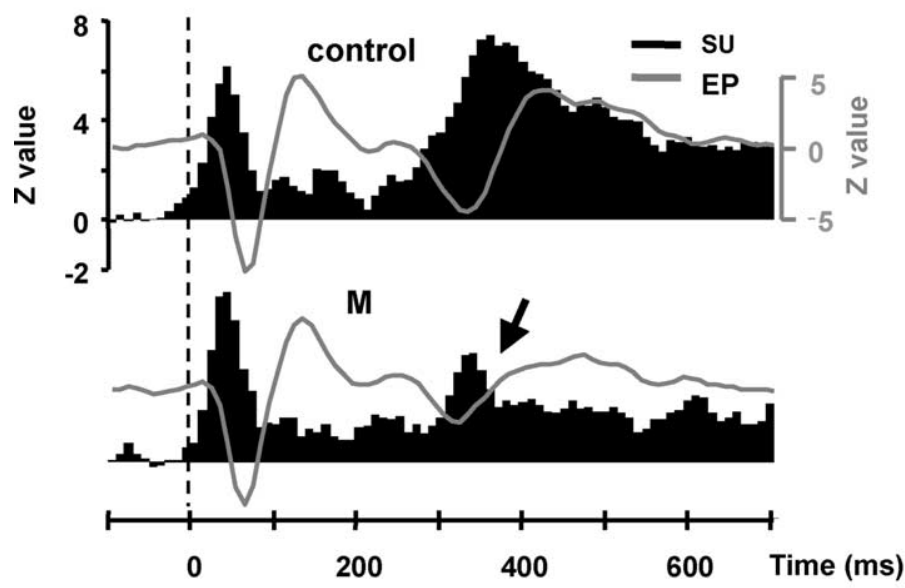

(b)

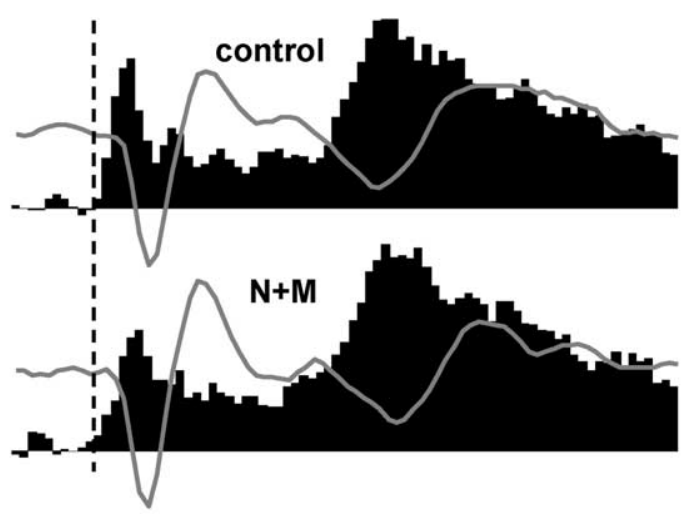

B

(a)

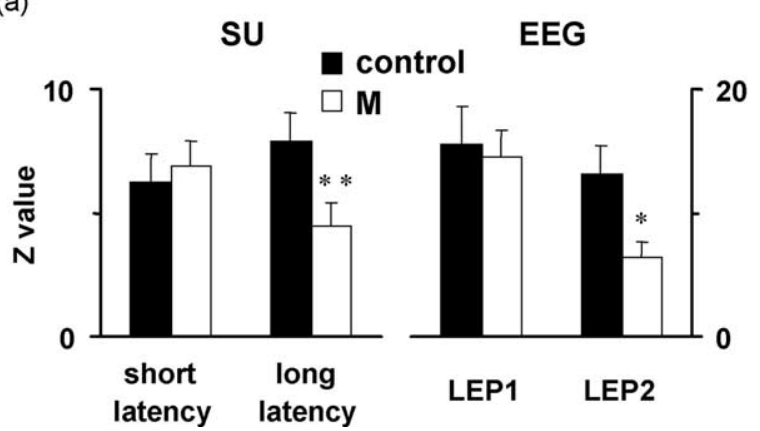

(b)

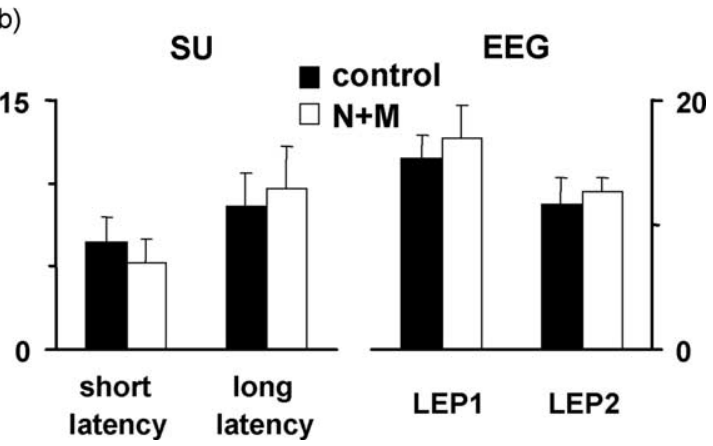

Fig. 6. Effect of intraperitoneally injected morphine $(10 \mathrm{mg} / \mathrm{kg})$ on laser heat-evoked responses of ensemble single-unit activity (SU) and EEG evoked potentials (EP) in the sensorimotor (SmI) tail region. (A) Pooled peri-event histograms of the effect of (a) intraperitoneal morphine (M), and (b) pretreatment with naloxone $(8 \mathrm{mg} / \mathrm{kg}$ ) followed by morphine $(\mathrm{N}+\mathrm{M})$. Note the markedly attenuated LEP2 and the long-latency evoked component of SU under morphine (arrow). Bin width, $10 \mathrm{~ms}$. (B) Paired $t$-test showed significant attenuation of LEP2 but not LEP1 (Ba, ${ }^{* *} p<0.01$; $* p<0.05$ ). This morphine effect was prevented by prior intraperitoneal administration of naloxone $(\mathrm{Bb})$. All data were normalized to $Z$ values of the 0.5 -s pre-stimulus period.

distributed responsive neurons may have contributed to the widely distributed A- $\delta$ and C-LEPs in the human and rat. On the other hand, the much-sharper and stronger responses of the homotypic SmI neurons may be used by the subject to precisely localize where the noxious stimuli have been applied.

\subsection{Preferential morphine effect}

We tested and provide direct support to the hypothesis that morphine preferentially suppresses the long-latency cortical LEP and unit responses. In contrast, the short-latency LEP and unit responses in the SmI were not significantly altered. Furthermore, all these morphine effects were reversible and were prevented by pretreatment with naloxone.

The analgesic effect of morphine is thought to be due to both spinal and supraspinal mechanisms. Electrophysiological experiments have demonstrated a preferential effect of systemic morphine on central nociceptive activity evoked by $\mathrm{C}$ nociceptors in anesthetized animal models. Carstens et al. (1979) found that lower doses of systemic morphine produced presynaptic inhibition on $\mathrm{C}$ inputs than those

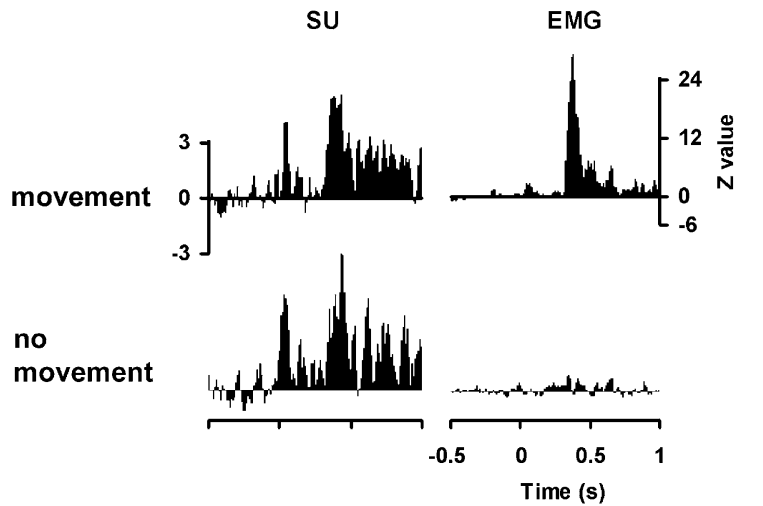

Fig. 7. Averaged peri-event histogram of multiple single-units (SU) in the primary sensorimotor cortex $(\mathrm{SmI})$ and electromyogram of the tail flick muscle (EMG) from three rats under $5 \mathrm{mg} / \mathrm{kg}$ of intraperitoneal morphine sulfate. About half $(48 \%)$ of the laser irradiation $(8 \mathrm{~W}, 15 \mathrm{~ms}$ at the midtail) evoked either a tail flick or a local movement (movement). These events were analyzed separately from those which evoked no outward movement (no movement). Note that in both groups, short-latency and long-latency SmI unit responses were elicited. 
needed on A- $\beta$ or A- $\delta$ primary afferent inputs. LeBars et al. (1980) and Johnson and Duggan (1981) showed that systemic morphine preferentially attenuated responses of dorsal horn nociceptive neurons to input from $\mathrm{C}$ fibers, and Jurna and Heinz (1979) described a preferential morphine effect on spinothalamic and spinoreticular cells to inputs from unmyelinated nociceptors. Bing et al. (1989) found a preferential effect of morphine on C-evoked responses of neurons in the subnucleus dorsalis of the medulla. Abdulla and Aneja (1993) also found that morphine inhibits the thalamic component of the subcortical somatosensory evoked potentials in rats. Matsumoto et al. (1987) demonstrated that the S-type tooth pulp-driven neurons (firing with a long-latency) of the SI of the cat are markedly suppressed after systemic morphine administration. This effect was completely reversed by naloxone. On the other hand, the discharges of F-type neurons that fired with a short-latency were not affected by morphine even under twice the dose that could suppress the S-type neurons. Kalliomaki et al. (1998) also showed that the laser-evoked long-latency cortical potential is blocked by intrathecal morphine treatment in halothane-anesthetized rats.

In sharp contrast to the electrophysiological data in anesthetized animals, there is only indirect evidence for a differential morphine effect on afferent inputs in awake humans and lab animals. Cooper et al. (1986) demonstrated that subjects trained to focus their attention on the magnitude of the first pain sensation evoked by a brief electrical or mechanical stimulation did not report a reduction by morphine. In contrast, the magnitude of second pain sensations produced by brief pulses of electrical, thermal, or mechanical stimuli in the same subjects was consistently attenuated. This suggests that morphine preferentially attenuates input from unmyelinated nociceptors. This hypothesis was reinforced by morphine's reduction of pain produced by capsaicin (presumably due to selectively exciting unmyelinated primary afferents) but its inability to diminish the pain elicited by bradykinin (presumably due to exciting $\mathrm{A}-\delta$ and $\mathrm{C}$ nociceptors). Yeomans et al. (1996) developed a monkey model to compare the effects of morphine on the first and second pain sensations. In human psychophysical experiments, brief (pulsed) thermal stimulation produced second pain sensations and ramp-and-hold thermal stimulation predominantly produced the first pain. The pulsed and ramp-and-hold forms of stimulation were used to test for differential effects of systemic morphine on the operant responses of the monkey. They found that low doses of morphine reduced operant responses to pulsed thermal contact, while higher doses were required to affect responses to ramp-and-hold thermal stimulation. Those results support systemic morphine preferentially attenuating second pain sensations.

Systemic morphine's attenuation of the long-latency nociceptive component but not the short-latency nociceptive component of cortical responses was demonstrated for the first time in the conscious behaving rats in the present study. The existing evidence suggests that activation of the peripheral nociceptors elicit complex processes in the cortical and subcortical structures. It is very likely that the cortical responses observed in the present study were the results of a cumulative action of morphine on many levels of the nociceptive pathway. Further SU recording experiments in conscious, unanesthetized conditions are necessary to reveal the neurophysiological mechanisms of the behavioral and psychophysical data.

\section{Acknowledgements}

This study was supported by the National Science Council, Taiwan (NSC91-2311-B002-017) and National Health Research Institute, Taiwan (EX90-9018EP).

\section{References}

Abdulla FA, Aneja IS. Morphine inhibits the thalamic component of the subcortical somatosensory evoked potentials in rats. Funct Neurol 1993;8:197-203.

Bing Z, Villanueva L, LeBars D. Effects of systemic morphine upon Aסand C-fibre evoked activities of subnucleus reticularis dorsalis neurons in the rat medulla. Eur J Pharmacol 1989;164:85-92.

Brink EE, Pfaff DW. Vertebral muscles of the back and tail of the albino rat (Rattus norvegicus albinos). Brain Behav Evol 1980;17:1-47.

Bromm B, Treede RD. Human cerebral potentials evoked by $\mathrm{CO}_{2}$ laser stimuli causing pain. Exp Brain Res 1987;67(1):153-62.

Carmon A, Mor J, Goldberg J. Evoked cerebral responses to noxious thermal stimuli in humans. Exp Brain Res 1976;25:103-7.

Carmon A, Dotan Y, Sarne Y. Correlation of subjective pain experience with cerebral evoked responses to noxious thermal stimulations. Exp Brain Res 1978;33:445-53.

Carstens E, Tulloch I, Zieglgansberger W, Zimmermann M. Presynaptic excitability changes induced by morphine in single cutaneous afferent C- and A-fibers. Eur J Physiol 1979;379:143-7.

Cooper BY, Vierck Jr CJ, Yeomans DC. Selective reduction of second pain sensations by systemic morphine in humans. Pain 1986;24(1): 93-116.

Johnson SM, Duggan AW. Evidence that the opiate receptors in substantia gelatinosa contribute to the depression, by intravenous morphine, of the spinal transmission of impulses in unmyelinated primary afferents. Brain Res 1981;207:223-8

Jurna I, Heinz G. Differential effects of morphine and opioid analgesics on $\mathrm{A}$ and $\mathrm{C}$ fibre-evoked activity in ascending axons of the rat spinal cord. Brain Res 1979;171:573-6.

Kalliomaki J, Weng HR, Nilsson HJ, Schouenborg J. Nociceptive C fibre input to the primary somatosensory cortex (SI). A field potential study in the rat. Brain Res 1993;622(1-2):262-70.

Kalliomaki J, Luo XL, Yu YB, Schouenborg J. Intrathecally applied morphine inhibits nociceptive $\mathrm{C}$ fiber input to the primary somatosensory cortex (SI) of the rat. Pain 1998;77:323-9.

Kenton B, Coger R, Crue B, Pinsky J, Friedman Y, Carmon A. Peripheral fiber correlates to noxious thermal stimulation in humans. Neurosci Lett 1980;17(3):301-6.

LeBars D, Guilbaud G, Chitour D, Besson JM. Does systemic morphine increase descending inhibitory controls of dorsal horn neurons involved in nociception? Brain Res 1980;202:223-8. 
Matsumoto N, Gotoh H, Sato T, Suzuki TA. Morphine selectively suppresses the slow response of tooth pulp-driven neurons in first somatosensory cortex (SI) of the cat. Neurosci Lett 1987;75: 55-9.

Mor J, Carmon A. Laser emitted radiant heat for pain research. Pain 1975;1: 233-7.

Nicolelis MAL, Ghazanfar AA, Faggin B, Votaw S, Oliveira LMO Reconstructing the engram: simultaneous multiple site, many single neuron recordings. Neuron 1997;18:529-37.

Paxinos G, Watson C. The rat brain in stereotaxic coordinates, 4th ed. San Diego, CA: Academic Press; 1998.

Shaw FZ, Chen RF, Tsao HW, Yen CT. Comparison of touch- and laser heat-evoked cortical field potentials in conscious rats. Brain Res 1999; 824:183-96.
Shaw FZ, Chen RF, Yen CT. Dynamic changes of touch- and laser heatevoked field potentials of primary somatosensory cortex in awake and pentobarbital-anesthetized rats. Brain Res 2001;911(2):105-15.

Tsai ML, Yen CT. A simple method for fabricating horizontal and vertical microwire arrays. J Neurosci Methods 2003;131:107-10.

Yen CT, Huang CH, Fu SE. Surface temperature change, cortical evoked potential and pain behavior elicited by $\mathrm{CO}_{2}$ lasers. Chin J Physiol 1994; 37:193-9.

Yen CT, Tsai ML, Kuo CC, Lee JC. Cellular basis of laser heat-evoked cortical potential studied with neuronal ensemble recording. 32 Annual Meeting, Society for Neuroscience; 2002.

Yeomans DC, Cooper BY, Vierck Jr CJ. Effects of systemic morphine on responses of primates to first or second pain sensations. Pain 1996; $66(2-3): 253-63$. 